\title{
Social Media Services and Technologies Towards Web 3.0
}

\author{
Neil Y. Yen • Chengcui Zhang • Agustinus Borgy Waluyo \\ James J. Park
}

Published online: 24 February 2015

(C) Springer Science+Business Media New York 2015

\section{Introduction}

Daily growth of the social media, together with numbers of service and application, reveals a significant change on information sharing. Unlike a conventional push-n-receive scenario, the social media offers a more attractive environment where the user acts as information transmitter and receiver - they create, comment, and remix new or existing information - and in other way adds extra value to the content in its ordinary form.

Although social media is a relatively new phenomenon, its roots can be traced to wellestablished and mature technologies and social behaviors that have underpinned information exchanges. What distinguishes social media, setting it apart from existing forms of online communication and interactions, is the ability of users to easily create and share information with their networks in real time, using not just computers, but also smart devices (e.g., mobile, sensors, etc.). This leads to a dramatic implication as information can reach wider audiences much faster, capturing their attention even when they are away from their computer screen. The success of online services such as Facebook, Twitter, and YouTube are testimony to the importance of social media and the impact they have had on both individuals and businesses.

Giving a quick glance to the history of Web, the fundamentals of information service explosion today was laid with Web 1.0, which is the read-only Web. The initial system created a constantly growing library for the publishing of information on static websites where users

\footnotetext{
N. Y. Yen

School of Computer Science and Engineering, University of Aizu, Aizuwakamatsu, Japan e-mail: neilyyen@u-aizu.ac.jp

C. Zhang

Department of Computer and Information Sciences, University of Alabama at Birmingham, Birmingham, AL, USA

e-mail: zhang@cis.uab.edu
}

\section{A. B. Waluyo}

Clayton School of Information Technology, Monash University, Melbourne, Australia e-mail: agustinus.borgy.waluyo@monash.edu

\section{J. J. Park $(\bowtie)$}

Department of Computer Science and Engineering, Seoul National University of Science and Technology, 172 Gongreung 2-dong, Nowon-gu, Seoul 139-743, South Korea

e-mail: jhpark1@seoultech.ac.kr 
can access it directly via browsers. Then, the Web 2.0 that we often consider it as the read-nwrite Web, or the Social Web in essence, makes it easy for the daily users to interact with, and further prompts them to participate in creating and publishing information as well.

Following the evidences, the Web 3.0 is envisioned to be associated with the evolution to an "Intelligent Web." It is anticipated that the Intelligent Web will address the lack of structure and organization in Web 2.0 era though linking the information from disparate sources and systems to ensure a more easy-to-use, efficient, and valuable scenario. It also implies a derivative of Semantic Web (or Knowledge Web) that interprets searchable content and thus delivers appropriate and relevant information according to the well understanding of the needs of users.

With more results coming up, we believe that a more appropriate term will be given to explain such promising and exciting phenomenon. But all in all, the evolution of Web is just in the beginning stage, and the Web updates itself with tremendously fast speed. New theories, techniques, and challenges will be discovered to best instance the phenomenon.

This special issue is taken as a pioneer to explore the meaning of Web 3.0. We totally received around twenty submissions from ten countries where the corresponding authors were majorly counted by the deadline for submission. All these submissions were found with significant contributions in the field of web research and social network analysis. But, however, only 12 high-quality papers were accepted after a two-round strict and rigorous review process. These accepted papers mainly look at our issue from the perspectives of social network analysis, knowledge discovery, big data mining, recommender system, multimedia, machine learning, information retrieval, security, human modeling, cognitive science and engineering, human-computer interaction, ubiquitous computing, e-learning, e-government, and their union, which brought lively discussions to the public readers.

\section{Summary of accepted papers}

The first paper entitled "Multi-Dimensional Attributes and Measures for Dynamical User Profiling in Social Networking Environments" by Xiaokang Zhou et al. [12] addresses a promising issue in regard to the individual information seeking process in an open social network. A universal user model, namely, Dynamically Socialized User Networking, is proposed to well describe multidimensional and correlated user characteristics and properties. A set of profiling and mining techniques are applied to analyze the shared information, such as posts, comments, etc., from users' social media. With the understanding of user behaviors on social media, this model is then applied to provide users, or coming users, with more adaptive information services that can better fit the needs of individual in their own social scenarios.

The second paper entitled "Digital Forensics Investigation Methodology Applicable for Social Network Services" by Yu-Jong Jang and Jin Kwak [7] considers the contexts, such as conversations, locations, and friendship relation, derived from individual social network for incident investigation. With the concern of differences between conventional applications and social network services, authors propose a digital forensic model specifically made for the use of social network services via smart devices. Three major steps, effective processing, digital device classification, and digital evidence collection and analysis, are included to ensure the security of digital data away from damage and manipulation.

The third paper entitled "Relative Weight Evaluation of the Factors Inducing Social Media Service Use" by Cheol-Rim Choi et al. [1] discusses the influence led by the explosion of social network service on the generation of business model, especially for the transformation of information. A set of mining techniques are designed to fairly examine the factors, i.e., 
social identity, altruism, telepresence, and perceptions, that may affect the usage situation of social network services and evaluate relative importance degree of each factor on the basis of relationships between with analytic network process.

The fourth paper entitled "Content Based Image Retrieval in a Web 3.0 Environment" by Aun Irtaza et al. [6] considers the importance of information retrieval, especially on the image aspect, in the Internet environment with a considerable number of multimedia applications. An image retrieval and management technique is proposed which focuses on the actual image contents without much dependency on their associated metadata (i.e., text-based annotations). This content-based retrieval technique incorporates genetic algorithms with support vector machines and user feedbacks to design the retrieval process, and assures the effective retrieval and sharing of images by taking the users considerations into an account.

The fifth paper entitled "User Tailored Cloud-Learning System using SNS and Learning Resources" by Hwa Young Jeong et al. [8] concentrates on the use of available resources over the existing social network services to develop a cloud-based personal learning system. A purpose-oriented matching mechanism is proposed to retrieve appropriate resources for the facilitation of self-regulated learning process. In addition, prompting the interactions, e.g., information sharing, discussions, etc., among participants through social network service also increase the learning motivation of involved users.

The sixth paper entitled "Adaptive Scheduling Approach for Game-Macth Arrangement and Schedule Optimization in Socially-Empowered Professional Sports Games" by Jason C. Hung et al. [5] attempts to formalize the scheduling problem for professional sports games, and further provides a solution to the issue of energy crisis. An adaptive scheduling algorithm is proposed to reduce the travelling costs of professional sports teams for NBA and MLB. In addition to finding an optimal schedule that meets all the restrictions, the fairness issue, say having balanced numbers of games at home and away, is addressed and the feasibility, as well as the performance, is also revealed by the real-world statistics.

The seventh paper entitled "Smart CDSS: Integration of Social Media and Interaction Engine (SMIE) in Healthcare for Chronic Disease Patients" by Iram Fatima et al. [3] points out the importance and emergence of social media, especially its use on prompting the interactions, in the development of clinical decision support system. An intelligent technique is proposed to extract the contexts from patients' social media, and label them with imperative location and semantic tags. All these outputs are applied in an implemented system, and help the health practitioners to understand the behavior and lifestyle of patients for better decisionmaking process.

The eighth paper entitled "Enhancing GMM Speaker Identification by Incorporating SVM Speaker Verification for Intelligent Web-Based Speech Applications" by Ing-Jr Ding and Chih-Ta Yen [2] focuses on the promising applications on the feature recognition over voice. Authors propose an EGMM-SVM model, that incorporates the Gaussian mixture method and support vector machine, to make improvement on accuracy of the estimated likelihood scores on existing Gaussian mixture method and quality transmission on support vector machine. The experiment reveals a higher recognition rate of newly proposed method than conventional one without any considerations on the quality of test speech utterances.

The ninth paper entitled "Game-based Image Semantic CAPTCHA on Handset Devices" by Tzu-I Yang et al. [11] focuses on the issues concerning the human cognitive process ability in an online social environment. Authors propose a new scheme that uses games to differentiate between humans and bots according to the game-based image semantics. This method is featured by its simple input, language-independent, high level in security, and enhanced ability on human cognitive process. Its feasibility is revealed by a series of empirical experiments on real-world testbed. 
The tenth paper entitled "Fast Video Encoding Algorithm for Efficient Social Media Service" by Kalyan Goswami et al. [4] looks at an issue in regard to the evolution of interactions among users and multimedia data on the Internet. This study proposes a new technique for early skip detection in order to reduce the time costs on encoding based on a well-designed statistical model. This method is implemented on HM 7.0 reference software and its significance in performance on time reduction is demonstrated with only a marginal quality loss.

The 11th paper entitled "Participatory Information Search and Recommendation Based on Social Roles and Networks" by Bo Wu et al. [10] indicates an issue concerning information explosion, both the information itself and its correlated network relationship, on the social network environment. Authors propose a recommendation service based on user's role and relationship. Beginning from the introduction of social role, a new term for the representation of social status of user, the core technique, participatory search, applied for formulizing the social relationship is then addressed to support the information seeking in an open social environment.

The last paper entitled "Watermark with DSA signature using Predictive Coding" by Cheonshik Kim and Ching-Nung Yang [9] then considers the issue on the enhancement of online security through the information hiding technique. Authors present a predictor-based watermark scheme that embeds secret bit streams and a DSA signature into an image. The proposed scheme conceals secret messages by using the divergence between current pixel values and predictive pixel values. Experimental results show that the newly proposed method receives low complexity and achieves a higher embedding performance with good perceptual quality compared to the conventional techniques.

\section{Conclusion}

The era of growing web and its evolution has begun, which promises to enhance the way information is retrieved, managed, and delivered, and thus provides better supports, in forms of services, applications, and so forth, to our daily lives. With the success in the organization of special issue on "Social Media Services and Technologies towards Web 3.0" in Multimedia Tools and Applications, it becomes possible for researchers (and interesting readers as well) who have been engaged in this or related areas to receive state-of-the-art information, gain experiences, and further bring about the benefits in this promising area of study. We, the guest editors, also envision the advanced stimulation of web evolution in the coming future.

Our special thanks go to Dr. Borko Furht, the Editor-in-Chief of Multimedia Tools and Applications, and all the editorial staffs for their supports throughout the preparation and publication process of this issue. We also thank for all authors who help a lot to advance this emerging field of study by submitting their outstanding research results. External and international referees are highly appreciated for their excellent contributions in reviewing the manuscripts.

\section{References}

1. Choi C-R, Jeong H-Y, Park J-H, Jeong Y-S (2014) Relative weight evaluation of the factors inducing social media service use. Multimedia Tools Appl. doi:10.1007/s11042-013-1713-4 
2. Ding I-J, Yen C-T (2014) Enhancing GMM speaker identification by incorporating SVM speaker verification for intelligent web-based speech applications. Multimedia Tools Appl. doi:10.1007/s11042-013-1587-5

3. Fatima I, Halder S, Saleem MA, Batool R, Fahim M, Lee Y-K, Lee S (2014) Smart CDSS: integration of social media and interaction engine (SMIE) in healthcare for chronic disease patients. Multimedia Tools Appl. doi:10.1007/s11042-013-1668-5

4. Goswami K, Kim B-G, Lee J-B, Jun D-S, Choi J-S (2014) Fast video encoding algorithm for efficient social media service. Multimedia Tools Appl. doi:10.1007/s11042-013-1728-X

5. Hung JC, Yen NY, Jeong H-Y, Chan Y-W (2014) Adaptive scheduling approach for game-match arrangement and schedule optimization in socially-empowered professional sports games. Multimedia Tools Appl. doi:10.1007/s11042-014-1852-2

6. Irtaza A, Jaffar A, Mutahira H, Muhammad MS (2014) Content based image retrieval in a web 3.0 environment. Multimedia Tools Appl. doi:10.1007/s11042-013-1679-2

7. Jang Y-J, Kwak J (2014) Digital forensics investigation methodology applicable for social network services. Multimedia Tools Appl. doi:10.1007/s11042-014-2061-8

8. Jeong HY, Hong BH, Park JJ (2014) User tailored cloud-learning system using SNS and learning resources. Multimedia Tools Appl. doi:10.1007/s11042-013-1717-0

9. Kim C, Yang C-N (2014) Watermark with DSA signature using predictive coding. Multimedia Tools Appl. doi:10.1007/s11042-013-1667-6

10. Wu B, Zhou X, Jin Q (2014) Participatory information search and recommendation based on social roles and networks. Multimedia Tools Appl. doi:10.1007/s11042-013-1849-2

11. Yang T-I, Koong C-S, Tseng C-C (2014) Game-based image semantic CAPTCHA on handset devices. Multimedia Tools Appl. doi:10.1007/s11042-013-1666-7

12. Zhou X, Wang W, Jin Q (2014) Multi-dimensional attributes and measures for dynamical user profiling in social networking environments. Multimedia Tools Appl. doi:10.1007/s11042-014-2230-9

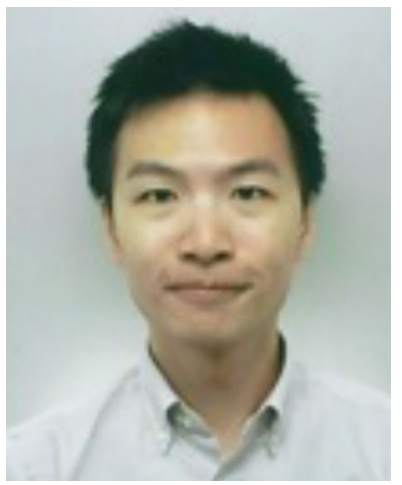

Dr. Neil Y. Yen received doctorates in Human Sciences at Waseda University, Japan, and in Engineering at Tamkang University, Taiwan in 2012. His doctorate in Waseda University was funded by the JSPS (Japan Society for the Promotion of Science) under RONPAKU program. He joins The University of Aizu, Japan as an associate professor since April 2012. Dr. Yen has been engaged extensively in an interdisciplinary field of research, where the themes are in the scope of Big Data Science, Computational Intelligence, and Human-centered Computing. Dr. Yen has been actively involved in the research community by serving as guest editors, associate editor and reviewer for international referred journals, and as organizer/chair of ACM/IEEE-sponsored conferences, workshops and special sessions. He is now a member of IEEE Computer Society, IEEE System, Man, and Cybernetics Society, and technical committee of awareness computing (IEEE SMCS). 


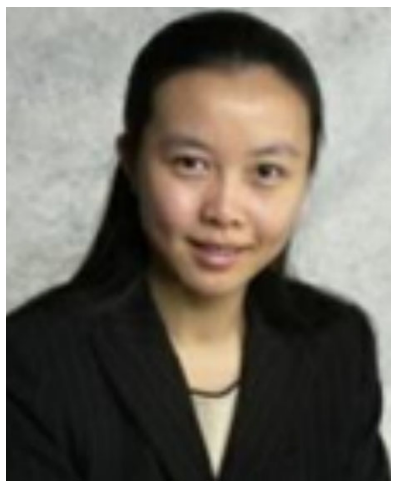

Chengcui Zhang (M'00) received the Ph.D. degree in computer sciences from Florida International University, Miami, FL, USA, in 2004. She is an Associate Professor with the Department of Computer and Information Sciences, The University of Alabama at Birmingham, Birmingham, AL, USA. She is the author and coauthor of over 130 research papers focusing on multimedia data mining, multimedia information retrieval, multimedia databases, bioinformatics, and geographic information systems. Prof. Zhang has served on more than 70 international conferences and workshops and taken a leadership role at the 2014 IEEE International Conference on Multimedia and Expo, the 2012-2013 IEEE International Conference on Information Reuse and Integration, 2012 IEEE International Symposium on Multimedia, and 2010 IEEE International Conference on Multimedia and Ubiquitous Engineering (MUE). In addition, she is a frequent panelist for National Science Foundation panels and an Editorial Board member of several international journals.

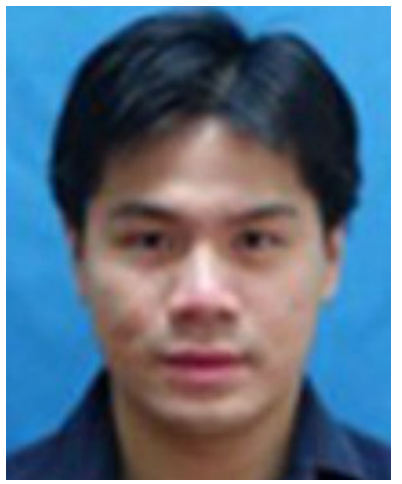

Agustinus Borgy Waluyo holds a Ph.D. in Computer Science from Monash University, Australia. Since the completion of his Ph.D. studies in 2006, he has involved extensively in large research and development projects. He was the lead member of the team and key developer of the projects, which resulted in several working prototypes. Waluyo's research expertise is revolved around the area of resource management and efficiency in wireless sensor networks, mobile query processing and optimisation, trust model and management, and data processing and analysis. 


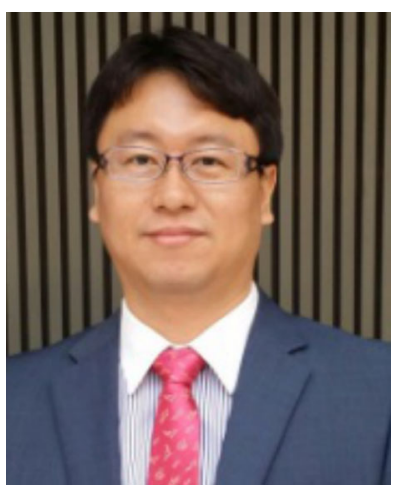

Dr. James J. (Jong Hyuk) Park received Ph.D. degrees in Graduate School of Information Security from Korea University, Korea and Graduate School of Human Sciences from Waseda University, Japan. From December, 2002 to July, 2007, Dr. Park had been a research scientist of R\&D Institute, Hanwha S\&C Co., Ltd., Korea. From September, 2007 to August, 2009, He had been a professor at the Department of Computer Science and Engineering, Kyungnam University, Korea. He is now a professor at the Department of Computer Science and Engineering and Deptartment of Interdisciplinary Bio IT Materials, Seoul National University of Science and Technology (SeoulTech), Korea. Dr. Park has published about 200 research papers in international journals and conferences. He has been serving as chairs, program committee, or organizing committee chair for many international conferences and workshops. He is a founding steering chair of some international conferences MUE, FutureTech, CSA, UCAWSN, etc. He is a president of the Future Technology Research Association International (FTRA) and Korea Information Technology Convergence Society (KITCS). He is editor-in-chief of Human-centric Computing and Information Sciences(HCIS) by Springer, International Journal of Information Technology, Communications and Convergence (IJITCC) by InderScience, and Journal of Convergence (JoC) by FTRA Publishing. He is Associate Editor/Editor of 14 international journals including 8 journals indexed by $\mathrm{SCI}(\mathrm{E})$. In addition, he has been serving as a Guest Editor for international journals by some publishers: Springer, Elsevier, John Wiley, Oxford Univ. press, Hindawi, Emerald, Inderscience. His research interests include security and digital forensics, Human-centric ubiquitous computing, context awareness, multimedia services, etc. He got the best paper awards from ISA-08 and ITCS-11 conferences and the outstanding leadership awards from IEEE HPCC-09, ICA3PP-10, IEE ISPA-11, and PDCAT-11. Furthermore, he got the outstanding research awards from the SeoulTech, 2014. Dr. Park' s research interests include Human-centric Ubiquitous Computing, Vehicular Cloud Computing, Information Security, Digital Forensics, Secure Communications, Multimedia Computing, etc. He is a member of the IEEE, IEEE Computer Society, KIPS, and KMMS. 